INPLASY

PROTOCOL

To cite: Guan et al. A systematic review and MetaAnalysis of Kuntai Capsule plus Western Medicines in patients with diminished ovarian reserve. Inplasy protocol 202190109. doi: 10.37766/inplasy2021.9.0109

Received: 30 September 2021

Published: 30 September 2021

Corresponding author:

Fang Lian

lianfangbangong@163.com

Author Affiliation:

Reproductive and Genetic

Center of Integtated

Traditional and Western

Medicine, Affiliated Hospital of Shandong University of

Traditional Chinese

Medicine.

Support: Foundation.

Review Stage at time of this submission: The review has not yet started.

Conflicts of interest:

None declared.

\section{A systematic review and Meta- Analysis of Kuntai Capsule plus Western Medicines in patients with diminished ovarian reserve}

Guan, L1; Zheng, M2; Wei, C3; Xiang, S4; Yu, Y5; Xin, X6; Lian, F7.

Review question / Objective: This study aims to evaluate the efficacy and safety of kuntai capsule (KTC) for the treatment of diminished ovarian reserve (DOR), either alone or in combination with western medicine.

Information sources: An electronic search of China National Knowledge Infrastructure (CNKI), WanFang, VIP, PubMed, and Cochrane Library databases was conducted to collect RCT reporting the use of the Kuntai capsule combined with western medicine for the treatment of ovarian reserve insufficiency. The search was conducted from inception to Feb,11,2021. The Chinese search terms included Kuntai capsules, ovarian reserve dysfunction, infertility, etc.; while the English search terms included, KTC, Infertility, Diminished ovarian reserve, etc.

INPLASY registration number: This protocol was registered with the International Platform of Registered Systematic Review and Meta-Analysis Protocols (INPLASY) on 30 September 2021 and was last updated on 30 September 2021 (registration number INPLASY202190109).

\section{INTRODUCTION}

Review question / Objective: This study aims to evaluate the efficacy and safety of kuntai capsule (KTC) for the treatment of diminished ovarian reserve (DOR), either alone or in combination with western medicine.

Condition being studied: Diminished ovarian reserve (DOR) refers to a decrease in the quantity and (or) the quality of 
oocytes in the ovary, antimüllerian hormone (AMH) levels, the number of antral follicle count (AFC), symptom score, and an increase in follicle-stimulating hormone (FSH) levels, and is associated with reduced fertility. In recent years, as the society advances, females are faced with double pressure from family and work responsibilities, substantially increasing the incidence of DOR, which may develop into ovarian failure within 1 6 years in the absence of any intervention. Therefore, it is extremely important to take effective measures to prevent and treat DOR. At present, the main treatment options used in DOR are hormone replacement therapy (HRT), ovulation induction therapy, and assisted reproductive technology (ART). However, HRT is associated with side effects that are likely to cause many complications, including an increase in the risk of breast cancer, especially following long time use. Despite substantial progress in ART, many failures result from decreased oocyte quality in patients with DOR. Currently, the treatment of DOR using Traditional Chinese Medicine (TCM) has attracted significant attention. Even though DOR is not identified in TCM, it is classified as a symptom of the delayed menstrual cycle, irregular menstrual periods, hypomenorrhea, amenorrhea, and infertility. According to the basic theory of TCM, the kidney stores essence, and is concerned with the development and reproduction of the body. Chinese medicine associates the main DOR mechanism to a deficiency of the kidney. Kuntai Capsule is a form of TCM based on a Huanglian Donkey-hide Decoction in "Treatise on Febrile and Miscellaneous Diseases" by the medical sage Zhang Zhongjing, and it comprises of Scutellaria baicalensis, Rehmannia glutinosa, Coptis, Peony, Ejiao, and Poria. Kuntai Capsule nourishes yin and nourishes blood, clears deficiency and heat, and soothes the nerves. In the prescription, Rehmannia glutinosa is the emperor's medicine, which is sweet and warm in action, and an indispensable medicine for nourishing kidney and essence, nourishing yin and blood. Rhizoma Coptidis, Radix Paeoniae Rubra, Donkey-hide Gelatin Nourish the Blood and
Nourish Yin, and they are compatible with Rehmannia glutinosa to enhance the nourishing effect of Rehmannia glutinosa. Scutellaria baicalensis Georgi and Poria cocos are adjuvants. Scutellaria baicalensis repels heat and relieves lung fire, strengthens the effects of Coptidis Coptidis in clearing heat and dampness, purging fire, and detoxification. Poria invigorates the spleen and nourishes the heart, soothes the heart and kidneys, provides a source of nourishing kidney essence, soothes the nerves, and treats deficiency and insomnia. These medicines work together to nourish the kidney and nourish the yin, clear the heat, and calm the nerves. Previous studies have found that one of the mechanisms of Kuntai Capsules is to prevent and treat low ovarian reserve by down-regulating FSH and LH levels, stimulating E2 secretion, upregulating $\mathrm{Bcl}-2$ protein expression, and down-regulating Bax protein expression to maintain the balance between apoptotic and inhibitory proteins and improve ovarian reserve. In addition, it plays an important role in treating DOR, by regulating the hypothalamus-pituitary-ovary axis, promoting follicle development, and improving the function of the ovary. However, most of the studies reporting the use of Kuntai Capsules in the treatment of DOR are single-center and with small sample size. Therefore, this systematic review and meta-analysis aimed to evaluate the efficacy and safety of KTC in DOR treatment, providing valid evidence for evidence-based medicine.

\section{METHODS}

Participant or population: Studies were included if (1)the study was a randomized controlled trial and semi-random controlled trial; (2) DOR was diagnosed according to Practical gynecological endocrinology (second edition) In western medicine and gynecology of Chinese medicine and separately in TCM. (3) the observation group used kuntai capsules with or without western medicine while the control group used western medicine.Exclusion criteria (1) Published articles were duplicates; (2) self-contrast before and after treatment; (3) 
case report; (4)animal experiments; (5)Nonrandomized controlled trial; (6) Repetitively published articles, choose one of them, and exclude the rest.

Intervention: The observation group used kuntai capsules with or without western medicine.

Comparator: The therapeutic intervention of controlled group was western medicine.

Study designs to be included: This review will include all available randomized controlled trials (RCTs) reporting the use of the Kuntai capsule combined with western medicine for the treatment of ovarian reserve insufficiency. Other studies such as case reports, reviews, retrospective studies, and studies using inappropriate random sequence generation methods will be excluded. The language limit is Chinese and English.

Eligibility criteria: The participant (P), intervention (I), comparator (C), outcome $(0)$, and study design (S) are the 5 main factors determining the inclusion and exclusion criteria of this research.

Information sources: An electronic search of China National Knowledge Infrastructure (CNKI), WanFang, VIP, PubMed, and Cochrane Library databases was conducted to collect RCT reporting the use of the Kuntai capsule combined with western medicine for the treatment of ovarian reserve insufficiency. The search was conducted from inception to Feb,11,2021. The Chinese search terms included Kuntai capsules, ovarian reserve dysfunction, infertility, etc.; while the English search terms included, KTC, Infertility, Diminished ovarian reserve, etc.

Main outcome(s): The primary outcomes included the overall effective rate and hormone levels (FSH, LH, E2).

Additional outcome(s): The secondary outcomes included FSH/LH, AMH, AFC, syndrome score and adverse reactions.
Data management: Study selection was performed by two independent researchers(Lu Guan and Mingming Zheng), if there were any disagreements, they were discussed and dealt with by a third researcher. All the studies obtained from the databases were imported into Endnote X9, and all the duplicates were eliminated. Data extraction was performed by reading the titles and abstracts, to select studies that met the inclusion criteria. Excel 2013 was used to extract the relevant information in the literatures, including: basic information (first author, publication time, country, etc.); baseline characteristics of the subjects (age, sample size, etc.); treatment methods and course of treatment of the control group and the experimental group; outcome index.

Quality assessment / Risk of bias analysis: Two trained researchers Chanfeng Wei and Shan Xiang will independently evaluate the risk of bias of the selected studies according to the Cochrane Collaboration's tool. In case of dispute, submit to corresponding author Fang Lian for arbitration. The risk of bias in included studies will be evaluated according to the following aspects: random sequence generation, allocation sequence concealment, blinding of participants and personnel, blinding of outcome assessors, incomplete outcome data, selective outcome reporting, and other sources of bias. Based on the above 7 entries, the judgment is divided into 3 levels: low risk of bias, high risk of bias, and unclear risk of bias. Results from these questions will be graphed and assessed using Review Manager 5.4.

Strategy of data synthesis: Meta-analysis was performed using RevMan5.4 software. Regarding the study outcomes, for binary variables, odds ratio (OR) or relative risk (RR) with $95 \%$ confidence interval $(\mathrm{Cl})$ were used, while for continuous variables, weighted mean difference (WMD) or standard mean difference (SMD) with $95 \%$ $\mathrm{Cl}$ were used. $\mathrm{P}<0.05$ indicated statistical significance. For testing the between-study heterogeneity in the results, the x2test was 
used (significance level: $P<0.1$ ). If the heterogeneity test demonstrated no heterogeneity $(P \geq 0.1,12<50 \%)$, a fixedeffect model was used. A random-effects model was used if there was heterogeneity $(P<0.1, I 2 \geq 50 \%)$; evidence of heterogeneity necessitated further subgroup analysis to determine the possible factors underlying the heterogeneity.

Subgroup analysis: If there is clinical and methodological heterogeneity, we will perform a subgroup analysis to explore the potential source of the heterogeneity according to the difference in interventions.

Sensitivity analysis: If necessary, sensitivity analysis will be used to assess the impact of the studies on the random effects model. After each study was excluded one by one, the data analysis was carried out again to determine the stability of the results.

Country(ies) involved: China.

Keywords: Kuntai capsule, Diminished Ovarian Reserve (DOR), Western medicine.

Contributions of each author:

Author 1 - Lu Guan.

Email: gl17862968201@163.com

Author 2 - Mingming Zheng.

Email: zhengmmzss@163.com

Author 3 - Chaofeng Wei.

Email: chaofeng_0415@163.com

Author 4 - Shan Xiang.

Email: axiangshan@163.com

Author 5 - Yi Yu.

Email: yuyi902016@163.com

Author 6 - Xin Xin.

Email: 2434059978@qq.com

Author 7 - Fang Lian.

Email: lianfangbangong@163.com 